\section{Triadisierung statt Globalisierung}

\author{
Aufgrund des hohen Niveaus und des zunehmenden Tempos grenzüberschreiten- \\ der ökonomischer Transaktionen wird verstärkt von einem Prozeß der Globali- \\ sierung oder gar einer globalen Ökonomie gesprochen. Eine genauere Betrach- \\ tung der empirischen Befunde zeigt aber deutlich, daß dies so nicht der Fall ist. \\ Geht es dabei im Kern nicht vielmehr um die Herausbildung weltwirtschaftli- \\ cher Blöcke?
}

Von Kurt Hübner

G lobalisierung ist ein auf vielen Ebenen stattindender Prozeß. Im Folgenden wird von einem eingeschränkten Globalisierungskonzept ausgegangen, dessen Untersuchung sich auf den ökonomische Kern in Form der weltweiten Beziehungen ökonomischer Prozesse konzentriert (1). Globalisierung meint dann entsprechend die Entstehung und Ausweitungen von Beziehungen zwischen ökonomischen und ökologischen Räumen in der Welt sowie den Einfluß, den sie wechselseitig aufeinander ausüben. Selbst ein derart eingeschränktes Konzept, das die kulturellen, sozialen und auch die politischen Beziehungen ausspart, birgt noch eine Reihe konzeptioneller und operationeller Probleme.

\section{- Handel mit Waren und Dienstleistungen}

Zwischen 1950 und 1992 hat das Volumen der Weltexporte im grenzüberschreitenden Handel mit Waren und Dienstleistungen mit einer jahresdurchschnittlichen (jds.) Rate von sechs Prozent sehr viel stärker zugenommen als das weltweite Bruttoinlandsprodukt (BIP). Die Exportsektoren, so die naheliegende Schlußfolgerung, sind wesentliche Träger des weltweiten ökonomischen Wachstumsprozesses. Unterstützt wird dieser von einer Vertiefung der weltweiten Arbeitsteilung sowie dem Aufbau globaler Tauschpunkte, die die nationalen ökonomischen Räume immer stärker miteinander verbinden. Diese Ergebnisinterpretation wird bestärkt, wenn man den Anteil der Exporte am Bruttoinlandsprodukt der Ökonomien innerhalb der OECD (Organisation für wirtschaftliche Zusammenarbeit und Entwicklung) als Indikator für den globalen Integrationsgrad einsetzt: Zwischen 1950 und 1993 ist für die Länder der OECD die Exportquote von durchschnittlich 8,4 auf 14,5 Prozent angestiegen.
Doch sollte dieser Befund nicht voreilig als Beleg für einen schnell steigenden globalen Integrationsprozeß angesehen werden. Differenziert man den Anstieg der Exportquote nach Volumengrößen (beispielsweise Tonnen) und Wertgrößen (beispielsweise US-Dollar), dann zeigt sich etwa für die Gruppe der OECD-Ökonomien, daß sich zwischen 1950 und 1990 das Volumen des Exports verdreifacht, aber die Wertgröße nur verdoppelt hat.

\section{Ausweifung ausländischer Direkt- investitionen}

Ein anderer häufig herangezogener Indikator der Globalisierung ökonomischer Systeme ist die Höhe der getätigten Direktinvestitionen ins Ausland. Seit den sechziger Jahren ist eine recht kontinuierliche Ausweitung ausländischer Direktinvestitionen zu beobachten. Mit den frühen Siebzigern und insbesondere nach der Überwindung der weltwirtschaftlichen Rezession von 1981/82 haben die ausländischen Direktinvestitionen stark an Dynamik gewonnen. Seither liegen deren jahresdurchschnittliche Wachstumsraten sogar weit über denen des Welthandels, der nationalen Bruttoinlandsprodukte und der weltweiten Bruttokapitalstockbildung:

- Die jds. Wachstumsrate an weltweiten Abfluissen ausländischer Direktinvestitionen betrug im Zeitraum 1981/93 knappe 15 Prozent. Der weltweite Bestand an Direktinvestitionen belief sich 1993 auf 2.135 Milliarden USDollar.

- Im gleichen Zeitraum stiegen die weltweiten Waren- und Nicht-Faktordienstleistungen jährlich um 5,9 Prozent an und

- die weltweite Bruttokapitalstockbildung verzeichnete ein Wachstum von 4,6 Prozent.

Getragen wurde diese bemerkenswerte Dynamik im Jahr 1994 mit 196 Milliarden US-Dollar mehr als 80 Prozent aller ausländischen Direkwesentlich von den OECD-Ökonomien, auf die tinvestitionen entfielen. Den Löwenanteil davon verbuchten mit 106 Milliarden US-Dollar die europäischen Volkswirtschaften.

Als Beispiele dieses Wachstums sollen im folgenden Japan, Großbritannien, Frankreich und die Bundesrepublik Deutschland gegenübergestellt werden: Während Japan im jährlichen Durchschnitt der Periode 1981/85 noch einen Direktkapitalexport von ,nur" fünf Milliarden USDollar (ohne reinvestierte Gewinne) aufzuweisen hatte, stieg er in der Periode 1986/90 auf einen jds. Betrag von 32 Milliarden US-Dollar an. In der Bundesrepublik hat sich dieser Wert im Vergleich zur Referenzperiode 1981/85 in der Periode 1986/90 vervierfacht, war aber insgesamt eher gering. Eine Verfünffachung wiederum weist Frankreich in diesem Periodenvergleich auf: der outflow von Direktinvestitionen betrug im Jahresdurchschnitt 1986/90 17 Milliarden US-Dollar, und war damit auch absolut betrachtet höher als im bundesdeutschen Fall. Und schließlich verdreifachten sich - ausgehend von einem hohen Niveau - die britischen Direktinvestitionen auf jds. 28 Milliarden US-Dollar in dieser Periode.

Alle diese Daten weisen zwar auf einen ausgeprägten Internationalisierungs- oder Globalisierungsprozeß der Produktionsstrukturen hin, allerdings erscheint eine gewisse Relativierung angebracht. Im Jahr 1992 hatten beispielsweise die gesamten outflows an ausländischen Direktinvestitionen gerade einmal einen Anteil von vier Prozent am weltweiten Waren- und Dienstleistungshandel von 4.545 Milliarden US-Dollar und der Anteil der Zuflüsse am weltweiten Kapitalstockaufbau machte in diesem Jahr gerade einmal durchschnittlich 3,7 Prozent aus. Und schließlich sollte nicht aus den Augen verloren werden, daß die outflows an Direktinvestitionen trotz ihres langen Wachstumsspurts in den OECD-Ökonomien nur in einigen wenigen Fällen mehr als ein Prozent am BIP ausmachten. Mit Ausnahme des Spezialfalles Großbritannien handelt es sich dabei durchweg um im ökonomietheoretischen Sinne kleine Volkswirtschaften wie die Schweiz, Schweden und die Niederlande. In den Volkswirtschaften mit den höchsten absoluten Beträgen wie den höchsten Zuwachsraten liegt der Anteil ausländischer Direktinvestitionen am BIP bei knapp einem Prozent.

Insgesamt zeigt sich also, daß die Entwicklungsdynamik von Welthandel und ausländischen Direktinvestitionen empirisch ein höchst exklusiver Vorgang ist. 


\section{- Triadisierung der globalen Ökonomie}

So entfallen heute etwa 60 Prozent des gesamten Weltexports von Gütern und Dienstleistungen auf die gleiche Gruppe von neun Industrieländern, die schon Mitte der sechziger Jahre die Spitzenpositionen einnahmen: Dies sind die USA, Kanada, Japan, Deutschland, Frankreich, Großbritannien, Italien, Niederlande und Belgien/Luxemburg. Das Bild läßt sich spezifizieren, wenn man die Welthandelsströme regional aufgliedert. Orientiert man sich an einem relativ weitgefaßten Regionalkonzept, dann zeigt sich eine ausgeprägte Triadisierung der Welthandelsströme.

Die sich aus den westeuropäischen und nordamerikanischen sowie aus den Ökonomien der asiatisch-pazifischen Region zusammensetzende Triade verbuchte Anfang der neunziger Jahre einen Welthandelsanteil von etwas mehr als 90 Prozent. Betrachtet man nur die weltweiten Exporte industriell gefertigter Güter, dann erhöht sich diese Quote sogar auf 93 Prozent. Allein der Handel zwischen den Triadenökonomien macht knapp drei Viertel des gesamten Welthandels aus.

Nach wie vor die wichtigste Welthandelsregion ist dieser Klassifikation zufolge Westeuropa, das einen Welthandelsanteil von 46 Prozent (1990) aufweist. Nordamerika (USA, Kanada) hat demgegenüber Anfang der achtziger Jahre seinen zweiten Platz an die Ökonomien des asiatischpazifischen Regionalblocks abgeben müssen, dessen Welthandelsanteil mittlerweile bei etwa 27 Prozent liegt. Für den ,Rest“ der Weltwirtschaft, insbesondere für die Gruppe der Entwicklungsländer, bedeutet dies einen stetigen Bedeutungsverlust. Sie haben an der beschleunigten Globalisierung gar nicht oder nur unter- proportional teil. Ihr Anteil am gesamten Welthandel geht bis Mitte der neunziger Jahre nicht über 15 Prozent hinaus. Innerhalb der letzten dreißig Jahre stellt dies einen herben Rückschlag dar. Anders stellt sich die Situation allerdings aus dieser - höchst heterogenen - Ländergruppe dar. Etwas mehr als zwei Drittel der gesamten Warenexporte dieser "Restkategorie“ fließen in die Triadenökonomien. Wenigstens ebenso exklusiv stellt sich die Verteilung der Direktinvestitionsbestände dar: Im Jahr 1993 konnte die Gruppe der Industrieländer nahezu drei Viertel aller Direktinvestitionsbestände auf sich vereinen.

\section{Grenzüberschreitende Geld- und Kapitaltransaktionen}

Seit den frühen siebziger Jahren haben die grenzüberschreitenden Geld- und Kapitaltransaktionen ein enormes Tempo- und Volumenwachstum erfahren. Der im Zuge des Zusammenbruchs des Weltwährungssystems von Bretton Woods vollzogene Übergang von einem System fixer zu einem Regime flexibler Wechselkurse hat wesentlichen Anteil an diesem Wachstum, wurde es doch jetzt für die privaten Akteure notwendig, sich gegenüber potentiellen Kursschwankungen durch entsprechende Kurssicherungsgeschäfte abzusichern. Die mit der Auflösung des Fixkurssystems verbundene Substitution des geldpolitisch getragenen Währungsschwankungsrisikos durch eine private Übernahme von Währungsrisiken induzierte entsprechend ein enormes Wachstum von Devisenmarkttransaktionen. Bis Mitte der neunziger Jahre gehen Schätzungen davon aus, daß der tägliche Umsatz an den wichtigsten weltweiten Devisenmärkten mehr als eine Billion US-Dollar beträgt.
Generell ist festzuhalten, daß Wachstum wie Volumen der grenziiberschreitenden Finanztransaktionen vor dem Hintergrund der aufgezeigten Globalisierungsprozesse keineswegs erstaunlich ist: Sowohl das hohe Wachstum des internationalen Handels mit Waren und Dienstleistungen als auch die beschleunigte Internationalisierung der Produktion via ausländischer Direktinvestitionen macht eine Ausweitung der internationalen Finanzströme unabdingbar. Bemerkenswert an dieser Entwicklung ist allerdings, daß das Volumen der internationalen Finanzströme sich seit mehr als einem Jahrzehnt von diesen monetären Unterfütterungsanforderungen emanzipiert hat und - in Bruttorechnung - das Volumen aus aggregierten weltweiten Handels- und Direktinvestitionsströmen weit übersteigt. Beispiel: Zwischen 1972 und 1992 hat das Volumen des Welthandels um etwa drei Billionen US-Dollar zugenommen; das Volumen internationaler Finanztransaktionen ist aber um etwa sieben Billionen US-Dollar angestiegen.

Dieses Wachstum läßt sich als Zunahme der internationalen finanziellen Tiefe interpretieren. Betrugen im Jahr 1964 die internationalen Nettoausleihungen des privaten Bankensystems gerade einmal 0,7 Prozent des Weltsozialprodukts, so stieg dieser Anteil bis 1980 auf immerhin acht Prozent, um dann bis 1991 auf mehr als 16 Prozent zu klettern. Dennoch kann nicht von einer Strangulierung der nationalen Räume und Akteure gesprochen werden. Nach wie vor gilt, daß ein - statistisch signifikanter - enger Zusammenhang zwischen nationaler Investitionsquotenentwicklung und Sparquotenentwicklung besteht und daß insbesondere die Ökonomien, die die Weltreservewährungen halten, über relativ ausgeprägte Freiheitsgrade in ihrer Geld- und Zinspolitik verfügen.

\section{Verteilung ausländischer Direktinvestitionsströme der Triade}

\begin{tabular}{|c|c|c|c|c|c|c|}
\hline \multirow[t]{2}{*}{ Zeitraum. } & \multicolumn{2}{|c|}{$1982-1985$} & \multicolumn{2}{|c|}{$1986-1989$} & \multicolumn{2}{|c|}{$1990-1992$} \\
\hline & Mrd. Dollar & Prozent & Mrd. Dollar & Prozent & Mrd. Dollar & Prozent \\
\hline Jährliche weltweite Ourflows & 42,2 & 100 & 153,5 & 100 & 199,5 & 100 \\
\hline Outflows der "EU der Zwölf" & 20,4 & 48,4 & 72,9 & 47,4 & 101,5 & 50,9 \\
\hline Oufflows der USA & 4,8 & 11,4 & 22,2 & 14,4 & 32,2 & 16,1 \\
\hline Oufflows von Japan & 5,0 & 11,9 & 28,1 & 18,3 & 32,0 & 16,0 \\
\hline Jâhrliche weltweite Inflows & 50,1 & 100 & 136,5 & 100 & 170,8 & 100 \\
\hline Inflows der "EU der Zwölf" & 13,1 & 25,9 & 49,2 & 36,0 & 83,6 & 49,0 \\
\hline Inflows der USA & 17,3 & 34,2 & 54,8 & 40,1 & 23,8 & 14,0 \\
\hline Inflows von Japan & 0,4 & 0,7 & 0,04 & 0,03 & 1,9 & 1,1 \\
\hline
\end{tabular}




\section{- Eine Interpretation der Befunde}

Von deutlich ausgeprägten ökonomischen Globalisierungsprozessen kann man also, wie die empirischen Befunde zu Handelsvernetzungen, Direktinvestitionsentwicklung, Geld- und Kapitaltransaktionen zeigen, vor allem seit den späten siebziger Jahren sprechen. Die Globalisierung erfolgt räumlich uneinheitlich, zeitlich geschichtet und in ihrem Tempo ungleichmäßig. Mit Blick auf das Tempo ist festzuhalten, daß die Ausweitung der globalen Produktionsbeziehungen ein weltwirtschaftlich gesehen herausragendes Ereignis darstellt. Vor allem infolge der Internationalisierung der Produktion und der Globalisierung der Wertschöpfungsketten bildet sich ein Verflechtungszusammenhang heraus, der die Standortproblematik nationaler Räume in engster Weise-berïhrt.

Natïrlich gilt auch: Globalisierung stellt sich im Kern als eine Triadisierung dar, als eine Herausbildung weltwirtschaftlicher Blöcke, auf die sich die Kernprozesse der grenzuiberschreitenden ökonomischen Transaktionen konzentrieren. Allerdings kann von ,einem“ Zustand der globalen Ökonomie freilich nicht gesprochen werden. Der globale ökonomische Raum ist vielmehr durch gleichzeitig wirkende Prozesse der Homogenisierung und Fragmentierung gekennzeichnet: Während sich der Verdichtungsgrad ökonomischer Praktiken innerhalb und zwischen den Triadeblöcken steigert, nehmen andere Regionen der Weltwirtschaft an diesem Vorgang fast gar nicht (Afrika südlich der Sahara) oder nur unterproportional teil (Ostmitteleuropa). Doch bedeutet Globalisierung andererseits für diese Regionen auch die Chance, an den sich intensivierenden grenzüberschreitenden Austauschbeziehungen teilhaben zu können, und damit auch die Hoffnung, sich im Zuge erfolgreicher Modernisierungsmaßnahmen einen - wenn auch kleinen - Teil des globalen Kuchens anzueignen.

\section{- Verschärfter Kampf zwischen den Akteuren der Triade}

Die auf der Ebene der Blöcke aufgezeigte ungleiche Struktur reproduziert sich auf der Ebene der Nationalökonomien: Während eine kleinere Zahl nationaler ökonomischer Räume in starkem Maße global ökonomisch ,verknotet“ ist, weisen eine große Zahl anderer nationaler Ökonomien nur lose Verknotungen auf. Enge Verknotungen weisen insbesondere die Kernökonomien der Triade auf. Sie verfügen im internationalen Vergleich über die höchsten Pro-Kopf-Einkommen und damit über die größten Volumina kaufkräftiger Nachfrage. Die Konzentration des Außenhandels und der ausländischen Direktinvestitionen auf diese Kerngruppe der globalen Ökonomie deutet darauf hin, daß es den Unternehmen in beiden Fällen primär darum geht, Anteile an dieser Kaufkraft zu erobern. Diese Beobachtung wird im übrigen auch von Motivanalysen für ausländische Direktinvestitionen bestätigt: Den Unternehmen geht es nach eigenem Verständnis in erster Linie um Marktanteile. Kostenaspekte spielen zwar auch eine Rolle, stehen aber nicht an erster Stelle.

Die nationalen Räume, deren spezifische Entwicklungsmodelle sowie die ökonomischen, politischen und sozialen Träger dieser Modelle werden durch Globalisierung dennoch unter den Streß von Anpassung und aktiver Restrukturierung gesetzt. Wenn von einer Intensivierung der Konkurrenz gesprochen wird, ist genau dieser Tatbestand angesprochen. Die Intensivierung der Konkurrenz ist dabei in erster Linie ein verschärfter Kampf zwischen Akteuren der Triade um Marktanteile, Wertschöpfungsanteile und damit um Einkommen und Arbeitsplätze.

Zentral für diese Form der Konkurrenz ist aber nicht vorrangig die Verbesserung der preislichen Wettbewerbsfähigkeit; im Vordergrund steht vielmehr der Ausbau der technologischen Wettbewerbsfähigkeit. Für diese Form der internationalen Konkurrenz stellen sich die trade-offBeziehungen zwischen ökonomischen und ökologischen Subsystemen aber prinzipiell günstiger dar als für den Fall der Kostenkonkurrenz.

\section{Anmerkung}

1) Die Ausführungen gehen auf ein Gutachten für die Enquete-Kommission "Schutz des Menschen und der Umwelt" unter dem Titel "Auswirkungen der Triebkräfte und Trends der Globalisierung auf eine Politik der Nachhaltigkeit" zurück, das der Autor zusammen mit dem IÖW erstellt hat. Eine Veröffentlichung ist für März/April 1997 von der Enquete-Kommission geplant.

\section{Der Autor}

Dr. Kurt Hübner ist Professor an der Fachhochschule für Wirtschaft, Berlin.

Kontakt: FHW, Badensche Str. 50-51, 10825 Berlin Tel. (030) $867-1$

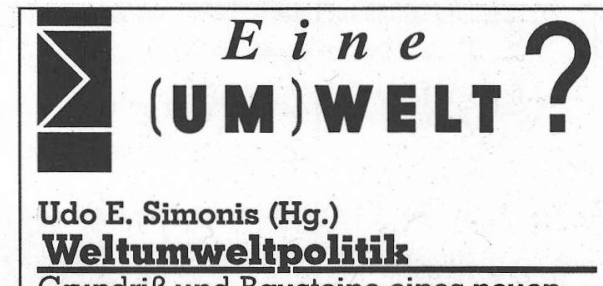

Grundriß und Bausteine eines neuen Politikfeldes

Die aktuelle Bilanz zum Stand und zu künftigen Aufgaben supranationaler Umweltpolitik 1996313 S. ISBN 3-89404-161-7 DM 36,00

Joseph Huber

Nachhaltige Entwicklung.

Strategien für eine ökologische und soziale Erdpolitik

Ein provokatives Plädoyer gegen simplifizierende und harmonistische Konzepte. 1995171 S. ISBN 3-89404-403-9 DM 27,80

Hans-Jürgen Harborth

Dauerhafte Entwicklung stat globaler Selbstzerstörung Eine Einführung in das Konzept des

"Sustainable Development "

Seit Jahren »die « Einführung par excellence.

${ }^{2} 1993134$ S. ISBN 3-89404-326-1 DM 19,80

Katz, Schmitt, Hennen, Sauter

Biotechnologien für die

„Dritte Weltu

Eine entwicklungspolitische

Perspektive?

Ist BioTech-Export Fluch oder Segen?

1996230 S. ISBN 3-89404-811-5 DM 36,00

Mickler, Engelhard, Lungwitz, Walker

Nach der Trabi-Âra:

Frbeiten in schlanken Fabrilken

Modernisierung der ostdeutschen

Autoindustrie

"Japanische Verhältnisse " im deutschen

Autobau: eine kritische Bestandsaufnahme

1996277 S. ISBN 3-89404-423-3 DM 39,00

Weiter i m Progra m m

Carsten Helm

Sind Freithandel und

Umwreltschutz vereinbar?

Ökologischer Reformbedarf des

GATT WTO-Regimes

1995180 S. ISBN 3-89404-151-X DM 29,80

Ānja Grothe-Senf, Ulf Kadritzke (Hg.)

Versöhnung von Öronomie

und Öliologie?

Theoretische und praktische Überprüfungen 1995 183 S. ISBN 3-89404-782-8 DM 19,80

Dieter Läpple ( $\mathrm{Hg}_{\mathrm{g}}$ )

\section{Gïterverkehr, Logistik} und Umwelt

Analysen und Konzepte zum interregio-

nalen und städtischen Verkehr

${ }^{2} 1995322$ S. ISBN 3-89404-352-0 DM 39,00

Unser Gesamtprogramm

kommt prompt und

unverbindlich zu Ihnen.

Postkarte/Anruf

genügt.

edition

sigmo

-Marx-Str. 17

12043 Berlin

Tel. [030] 6232363

Fax 6239393 
(c) 20I0 Authors; licensee IÖW and oekom verlag. This is an article distributed under the terms of the Creative Commons Attribution Non-Commercial No Derivates License (http://creativecommons.org/licenses/by-nc-nd/3.o/), which permits unrestricted use, distribution, and reproduction in any medium, provided the original work is properly cited. 